\title{
Airwake characteristics simulations with different turbulence models
}

\author{
Gao Zhe-Ming ${ }^{1, a}$,Liu Chang-Meng ${ }^{2, b}$ \\ ${ }^{1}$ Harbin Engineering University, Harbin 150001,China; \\ ${ }^{2}$ The 41st Institute of the Fourth Academy CASC, Xi'an 710025, China. \\ amicroming01@sina.cn, bliuchangmeng2126@126.com
}

Keywords: Computational fluid dynamics, Numerical simulation, Airwake, Frigate.

\begin{abstract}
Characteristics of flow field on the flight deck for the frigate ship are complex. In order to find an accurate and worthy method to simulate the ship airwake, isolated simplified frigate ship, SFS2, was calculated by CFD(Computational Fluid Dynamics). The different turbulence models, SKE, MMK and DES, were used to simulate the flow field. CFD results were used to analyze the characteristics of flow field around the flight deck through compared with the experimental data in wind tunnel. The existence of horseshoe vortex on the surface of the flight deck was verified. Vortices separated off the top of the hangar and reattached on the flight deck. The results show that the DES model calculation results are more accurate than SKE and MMK model, which is suitable for engineering applications.
\end{abstract}

\section{Introduction}

To date, High fidelity piloted flight simulation could be used to provide a much safer alternative to sea trials and give the added benefit of allowing investigative flight tests for new ships still at the design stage[1].The literature shows various studies of the ship airwake with different numerical tools. Tai and Carico[2] used steady Reynolds Averaged Navier-Stokes (RANS) scheme solvers to calculate mean flow characteristics of airwake about a simplified DD-963 ship. Later, Tai[3] utilized a multi-zone, thin-layer Navier-Stokes method to provide the airwake about an LHD ship configuration. Applied to this frigate shape on structured grids have been steady Navier-Stokes solvers by Reddy et al.[4]. A very detailed analysis of a landing helicopter assault ship using an unsteady Navier-Stokes solver was carried out by Polsky[5]. The LHA CFD simulations show good agreement with the results of wind tunnel test.

In the present work, CFD was used to calculate the air flow through the isolated ship deck airflow field. To perform better in the shear flow field, the modified model, MMK $k-\varepsilon$ (MMK) model[6] and DES, were introduced to calculation by the user defined functions (UDF) interface. The objective was to build an approach to perform simulations on coarse grids with low computational cost. The results show that the MMK and DES can reflect the characteristics of the flow field, which can be better used in engineering calculations.

\section{Simulation method}

CFD model. In the present work, a simple frigate model of the international generic was calculated (SFS2, Fig. 1). The main vortex features of this model were similar to the structure of the flow field generated by a typical frigate. Numerous researchers from USA, UK and Canada used this model for parallel numerical simulation, wind tunnel and water tunnel experiments. Specific size parameters of the model SFS2 were seen in the literature [7].

Fig. 1(b) is a frigate CFD model. As much as possible to retain the integrity of a ship structure, CFD model made some necessary simplification based on the limit of CFD calculation. The model SFS2 is closer to the real frigate. Airflow field research on SFS2 can help to find the essential characteristics of the frigate ship structure without the interference of the flow field generated by the entity ship antenna and chimney structure. 


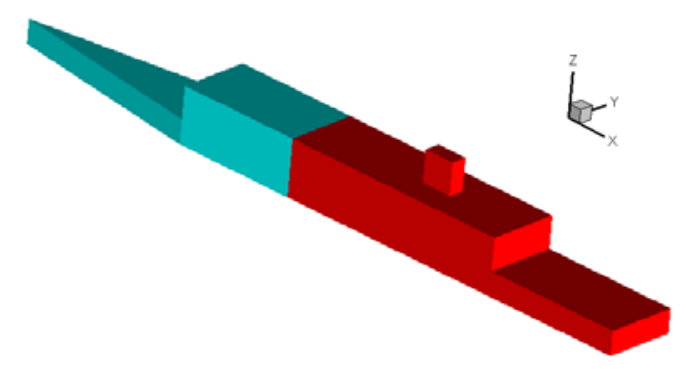

Fig. 1 CFD geometry

Grid generation. The calculated grid was generated by the ANSYS ICEM (Fig. 2). Due to relatively simple SFS2 geometry, full structured grid was used. Mesh was refined in the near-wall region. The entrance of $20 \mathrm{~m} / \mathrm{s}$ wind speed case, the first layer grid height is set as $0.005 \mathrm{~m}$ to guarantee to meet $y+$ conditions of the turbulence model using wall functions.

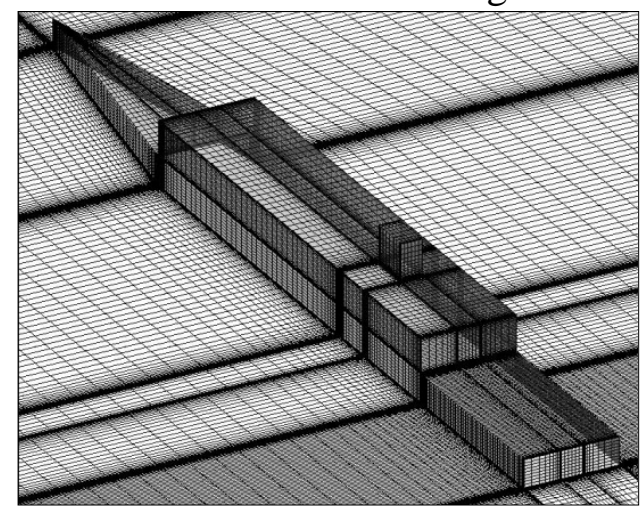

Fig. 2 Details of the ship grid

Flow Solver and calculation settings. The incompressible flow was simulated with a pressure-based type here. The low velocity flow was essentially incompressible. For this study, cases were computed to simulate full scale conditions. The full-scale ship solutions were run on a high-performance IBM computer cluster using 160 processors with unsteady model. The time step was set to 0.002 seconds based on free flow speed and the smallest grid spacing.

All calculations were performed with second order accuracy in time and space. The damping parameters were applied for code dependent.

\section{Results and discussions}

Ship airwake flow topology. SFS2 showed the flow of the front steps at the bow, followed by the flow phenomena of the backward-facing step at the flight deck and hull tail. The flow structure around the flight deck was the most common three-dimensional flow phenomena with the backward facing step flow. The flow chart at the SFS2 middle section of the flight deck is in Fig. 3. The air shows the shear layer separated at the top of the hangar and reattached on the flight deck. There is a huge recirculation zone behind of the hangar.

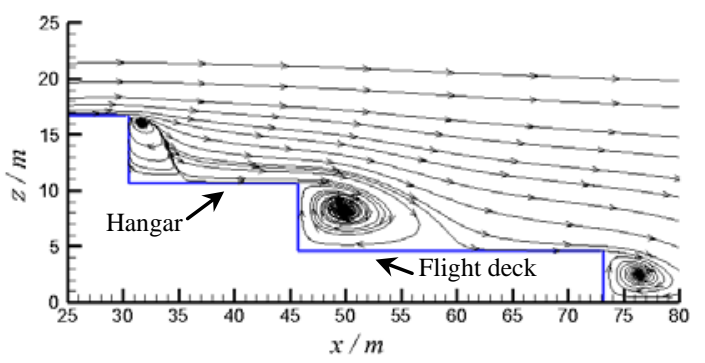

Fig. 3 Two-dimension streamlines in the middle section of the flight deck

Fig. 4 shows a horseshoe vortex captured behind the hangar wall. Compared with the literature [8] in the wind tunnel experiment picture, calculation of this paper showed accurately the location of the horseshoe vortex. In the vortex area near the wall of the flight deck, a horseshoe-shaped shear layer is in existence. While in the horizontal direction, due to blocking of the hangar for the fluid, 
airflow behind the hangar generated two symmetric reflux. The flow field structure was quite complex behind the hangar and presented a strong three-dimensional features. It can be seen that the results show good agreement with experimental data to validate the CFD method.

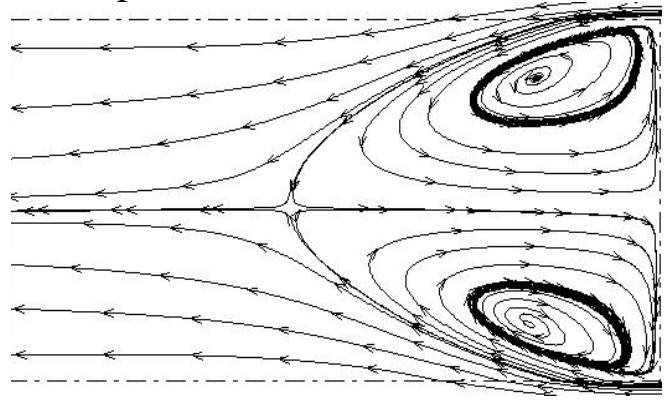

Fig. 4 Surface streamlines on flight deck of SFS2

Resuits with different models. Because the sharp corner of a bluff body is a problem point in CFD, it is very difficult to analyze accurately the flow field behind a sharp corner. Due to lack of RANS turbulence models, the problem is not solved struck whatever fine grids are used. At present, there is no effective way for avoiding the problem. It should be very careful when dealing with the flow field around a sharp corner.

The MMK model and DES with good application in the field of wind engineering was introduced to simulate accurately the ship deck airwake. The results were expected to can provide an important reference for engineering calculation of the fluid dynamics and establish a best design method early in a ship's planning stage.

It can be seen, in Fig. 5, the CFD calculation in this article results show good agreement with the wind tunnel experimental results. The overall distribution trend of the velocity curve is symmetrical. However, the starboard speed is slightly smaller than the larboard side of the speed distribution from the experimental data with $u$ direction component, which presents a weak asymmetry. Both sides of $v$ direction velocity component provide the opposite direction. Lateral air from the deck edge should be extruded to the deck center. Velocity magnitude presents a good symmetry. The significant downwash in $w$ direction also shows that the downward movement of the separation of the airflow at the top of the hangar attaches to the flight deck.

The calculation results show that the three models provide a good show with correct velocity distribution trends. But the MMK model and DES model calculation results are better than SKE model. The data show that the DES calculation results are more accurate compared to the MMK calculate results in the $u$-direction. DES model demonstrated the computational advantages.

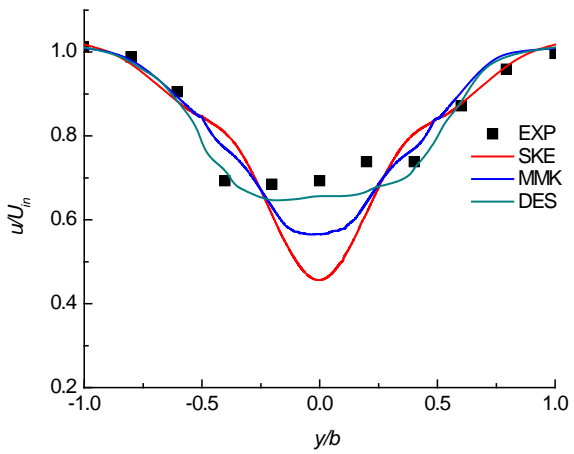

(a) Longitudinal velocity distribution

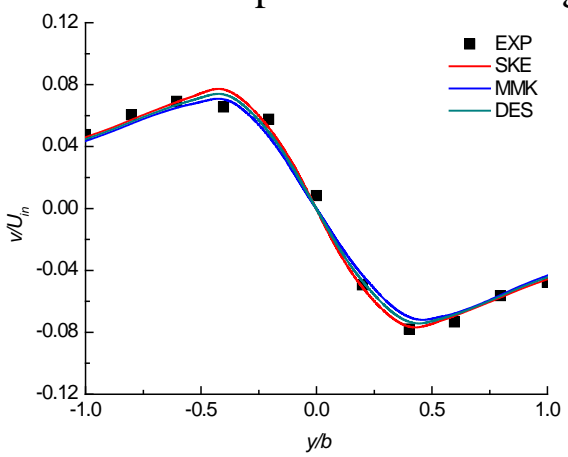

(b) Transverse velocity distribution 


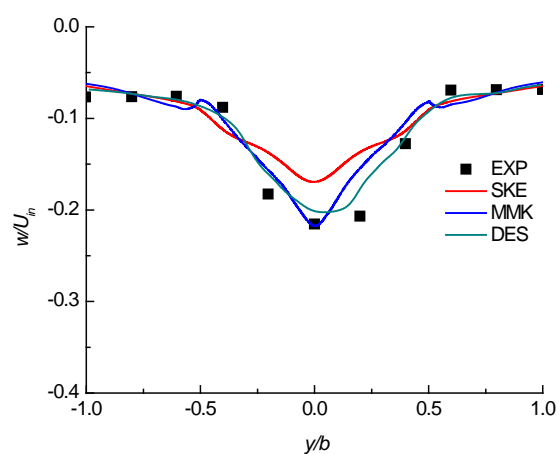

(c) Vertical velocity distribution

Fig. 5 Mean velocities normalized by $U$ at $50 \%$ flight deck length

\section{Summary}

The field structure of airflow is complex around the frigate flight deck, but still has a stable vortex structure and flow characteristics. DES and MMK are able to capture the most dominant flow while SKE only gives a only a poor representation of the unsteady flow phenomena. DES is superior to SKE and MMK. DES model has the computational advantages to simulate the ship airwake in wind engineering.

\section{Acknowledgments}

This paper is supported by the National Science Foundation of China under Grant No. 115720 9 5. The corresponding author is Gao zhe-ming, (1989- ), male, $\mathrm{PhD}$ candidate, E-mail: micro-ming01@sina.cn.

\section{References}

[1] M.C. Bogstad, W.G. Habashi, I. AKEL, Computational fluid dynamics based advanced ship airwake database for helicopter flight simulators, J. Aircraft, 2002, 39(5): 830-834.

[2] T.C. Tai, D. Carico, Simulation of DD-963 Ship Airwake by Navier-Stokes Method, J. Journal of Aircraft, 1995 32(6): 1399-1401.

[3] T.C. Tai, Simulation and Analysis of LHD Ship Airwake by Navier-Stokes Method. RTO AVT Symposium. Amsterdam, 1998,

[4] K. Reddy, R. Toffoletto, K. JONES, Numerical simulation of ship airwake, J. Comput \& Fluids, 1999, 29: 451-465.

[5] S.A. Polsky, W.S.B. Christopher, Time-Accurate Computational Simulations of an LHA Ship Airwake. 18th Applied Aerodynamics Conference, Denver, USA, 2000.

[6] M. Tsuchiya, S. Murakami, A. Mochida, K. Kondo, Y. Ishida, Development of a new $k-\varepsilon$ model for flow and pressure fields around bluff body, J. Journal of Wind Engineering and Industrial Aerodynamics, 1997, 67: 169-182.

[7] D.M, Roper, I. Owen, G.D. Padfield, S.J. Hodge, Integrating CFD and piloted simulation to quantify ship-helicopter operating limits, J. Aeronaut, 2006, 110(1109): 419-28. 